\title{
La carga mental de los profesionales de Enfermería en relación con su turno laboral
}

\author{
Laura Almudéver Campo ${ }^{1}$ \\ Iris Pérez Jordan ${ }^{2}$
}

1.- Profesora asociada Facultad de Enfermería y Podología. Universidad de Valencia, España.

2.- Grado Enfermería. Universidad de Valencia.

Recibido: 05 de mayo de 2018

Aceptado: 02 de febrero de 2019

\section{Resumen:}

La carga mental es el conjunto de requerimientos mentales, cognitivos o intelectuales a los que se ven sometidos los trabajadores a lo largo de su jornada laboral. El presente estudio pretende conocer la prevalencia de los niveles de carga mental en profesionales de Enfermería de varias unidades de hospitalización de un hospital, así como su relación con el turno de trabajo y otros factores sociodemográficos y laborales. Para ello se ha realizado un estudio cuantitativo de carácter descriptivo, prospectivo y transversal. Los resultados nos indican que un 
$55 \%$ de los enfermeros tienen un nivel de carga mental medio y más del $40 \%$, un nivel alto.

Palabras clave: carga de trabajo; enfermero; trabajo;

\begin{abstract}
:
The mental load is the set of mental, cognitive or intellectual requirements to which workers are subjected during its working hours. The present study aims to know the prevalence of mental load levels in nurses of several hospital units, as well as its relationship with the workshift and other socio-demographic and labor factors. It has conducted a quantitative, descriptive, prospective and cross-sectional study. From the results obtained, has been detected that $55 \%$ of those nurses have a medium level of mental load and more than $40 \%$, a high level.
\end{abstract}

Keywords: workload, nurse, work.

\title{
1. Introducción
}

La realidad laboral viene determinada históricamente por factores socioeconómicos, los cuales involucran en la actualidad modelos de producción y prestación de servicios continuos y acelerados (De Cássia et al, 2014). Dichos modelos seguidos por las organizaciones, obligan a los trabajadores a someterse a horarios de trabajo que van en contra del ritmo natural de la vida y que tienen una repercusión directa sobre la salud, entendiéndose ésta como un completo bienestar físico, mental y social, y no solamente la ausencia de afecciones o enfermedades (WHO, 2016). 
Podemos definir 'carga mental' como el conjunto de requerimientos mentales, cognitivos o intelectuales a los que se ve sometido el trabajador a lo largo de su jornada laboral, es decir, el nivel de actividad mental o de esfuerzo intelectual necesario para desarrollar su trabajo (Sebastián y Del Hoyo, 2002). Esta carga viene determinada por la interacción que se establece entre las exigencias del trabajo, incluyendo las condiciones en las que se realiza, y las características del individuo. Dicha carga se puede evaluar mediante medidas de rendimiento, técnicas subjetivas, registros psicofisiológicos e incluso técnicas analíticas.

El sector sanitario y más concretamente el de la Enfermería convive constantemente con condiciones laborales basadas en el trabajo por turnos de entre 7 a 12 horas, rotando en días y noches para que haya un reparto equitativo de turnos nocturnos entre los trabajadores de un mismo hospital o servicio. Normalmente existen tres turnos de trabajo diferentes: matutino (de 8 a 15 horas), vespertino (de 15 a 22 horas) y nocturno (de 22 a 8 horas), aunque también existen turnos de 12 horas en los que una misma persona trabaja de las 8 a 20 horas o de 20 a 8 horas del día siguiente (NIOSH, 2016). Algunos estudios han comparado los turnos de 12 horas y los de 7 horas, y las ventajas del más extenso parecen varias, como menores niveles de estrés, mejores condiciones físicas y psicológicas, una mejora del bienestar y de la calidad del sueño, así como de las relaciones sociales (Moreno et al, 2013).

Además de la duración del turno, es importante conocer en qué momento del día se lleva a cabo éste, si matutino, vespertino o nocturno, pues no todos suponen el mismo reto para la salud del individuo (Morin, 1970). Cuando un individuo debe trabajar en un turno que coincide con las horas de sueño de su ritmo circadiano (horario nocturno), es probable que se encuentre adormecido. Por el contrario, cuando trate de conciliar el sueño durante el día al salir del trabajo, puede encontrarse con dificultades y no descansar bien, pues estará en sus horas activas del ritmo (Moreno et al, 2013). Este tipo de alteraciones repetidas en el tiempo, terminan derivando en numerosos problemas de salud. 
Sin embargo, existen otros aspectos que también influyen en el trabajador y en su carga mental, como la duración del turno, el número de turnos antes de un día libre, el número de días de descanso en un fin de semana, el número de horas extra trabajadas, el tiempo de descanso entre turnos, el tiempo de descanso durante el turno y la regularidad y previsibilidad del calendario laboral.

La suma de todos estos aspectos es la que determina si las condiciones de trabajo son favorables o desfavorables para los trabajadores, afectando al nivel de estrés y a la calidad de vida del individuo, sobre todo cuando las horas trabajadas son demasiadas, interrumpen los ritmos naturales del ser humano y se tienen escasos descansos. Todo ello podría suponer una reducción de la calidad y seguridad asistencial de los pacientes, pues enfermeros bajo estas circunstancias pueden desarrollar de forma ineficiente sus actividades, resultando en consecuencias negativas al individuo o a la población asistida (De Cássia et al, 2014).

Los principales efectos negativos en la salud de los trabajadores con turnos largos y rotatorios, son aquellos relacionados con la calidad del sueño, el estrés y la fatiga, pero mantenidos en el tiempo se manifiestan en numerosos síntomas y patologías.

Algunos estudios apuntan a que el 50\% de los trabajadores nocturnos tienen problemas de sueño, mientras que en el caso de individuos con turnos rotativos, el porcentaje asciende al 66\%. Por el contrario, los trabajadores diurnos fijos apenas oscilan entre el 5,2\% y el 11\% en cuanto a problemas de descanso (Feo, 2007). Estos problemas suelen estar relacionados con dificultad para conciliar el sueño, interrupciones frecuentes del sueño, sensación de no haber tenido un descanso de calidad y somnolencia (Tovalin, 2005).

Los trabajadores nocturnos también ven afectado el horario y la secuencia de sus comidas, pues en plena noche el estómago no está acostumbrado a digerir comidas pesadas y puede producirse inapetencia y cambios en los ritmos de eliminación. Al no producirse una alimentación regular y eficaz en cantidad ni calidad, se produce estreñimiento, crisis hemorroidales o falta de apetito entre otros (Knauth, 2001; Feo, 2007). 
Los cambios en los horarios traen consigo efectos negativos para la salud reproductiva de la mujer trabajadora, incrementándose el riesgo de padecer infertilidad, problemas para quedarse embarazadas, abortos y problemas en los recién nacidos. Por otro lado, la alteración del ritmo circadiano y un elevado nivel de estrés desencadenan en una disminución de la capacidad de concentración, anergia, apatía, irritabilidad, trastornos del sueño y del apetito, sensaciones paranoides de ser objeto de críticas y desesperanza (Feo, 2007).

Por todo ello, el objetivo principal de esta investigación se centra en conocer la prevalencia de la carga mental en los profesionales de Enfermería, así como la relación de ésta con el turno laboral y otros factores sociodemográficos y laborales. De forma más específica, los objetivos que se pretenden perseguir son:

Medir el nivel de carga mental de los enfermeros de diferentes unidades de hospitalización de un hospital.

Comparar el nivel de carga mental de los profesionales de Enfermería del estudio según su turno de trabajo.

Comparar el nivel de carga mental de los enfermeros según factores sociodemográficos y laborales como la edad, el sexo, la tenencia de hijos, la antigüedad del puesto de trabajo y el tipo de contrato.

\section{Material y métodos}

Tipo de estudio: Se trata de un estudio descriptivo, prospectivo y transversal, de diseño cuantitativo.

Ámbito de estudio: Unidades de Neurocirugía-Otorrinolaringología, Neurología, Neumología y Medicina Interna-Unidad de cuidados continuados del 
Hospital General Universitario de Valencia. Todas ellas cuentan con una plantilla media de 12 profesionales de Enfermería en cada unidad. El ratio enfermera/paciente suele estar entre 10 y 15 pacientes por profesional de Enfermería en cada turno, y los pacientes se encuentran ubicados en habitaciones de 2 o 4 personas.

Periodo de estudio: Este estudio se llevó a cabo entre los meses de noviembre de 2016 a mayo de 2017.

Criterios de inclusión: Se consideraron criterios de inclusión que el sujeto de estudio fuese enfermero de las unidades de hospitalización nombradas anteriormente y que ejerciese dicha profesión sanitaria al menos 1 año.

Criterios de exclusión: Enfermeros que ocupan un puesto en alguna de las unidades nombradas durante un solo turno como caso excepcional.

Mecanismo de selección de la muestra: La selección de los profesionales de Enfermería para el estudio se llevó a cabo mediante un muestreo de conveniencia.

Tamaño muestral: La muestra estuvo formada por un total de 40 profesionales de Enfermería, 20 de ellos con un turno de 7 horas y los 20 restantes, con uno de 12 horas.

Mediciones: Para medir el agotamiento mental se ha utilizado el cuestionario NASA-TLX, que es un método de valoración de la carga de trabajo de forma subjetiva por parte de los sujetos que lo responden. Al tratarse de un 
instrumento de valoración de carácter subjetivo, está adaptado de forma que es sensible a las variaciones dentro y entre tareas, con capacidad de diagnóstico sobre las fuentes de carga, y relativamente insensible a las variaciones interpersonales.

Se parte del supuesto que la carga de trabajo es un concepto hipotético relacionado con los niveles de rendimiento. Dicha carga se mide mediante las exigencias de la tarea, que puede verse modificada por una serie de variables, tres de ellas haciendo referencia a la demanda impuesta sobre la persona y otras tres a la interacción de la persona con la tarea. Para poder aplicar el NASA-TLX, hay que tener en cuenta dos fases, una de ponderación y otra de puntuación.

Durante la fase de ponderación, el sujeto a estudio determina el grado en que cada una de las seis dimensiones propuestas por el cuestionario contribuye a la carga en cada tarea o subtarea específica. Las dimensiones se agruparán en pares para después estudiar el elemento de cada par que dé la fuente más significativa de variación de carga de trabajo en estas tareas. Estos pares son F-M, T-M, R-M, Fr-M, E-M, T-F, R-F, Fr-F, E-F, T-F, T-R, T-Fr- T-E, R-Fr, R-E y E-Fr. Estas dimensiones son exigencia mental, física, temporal, esfuerzo, rendimiento y nivel de frustración, y se les puede dar un peso de 0 (mínimo) a 5 (máximo) puntos según las elecciones de cada factor en los pares (Tabla 1).

Tabla 1.

DIMENSIÓN EXTREMOS DESCRIPCIÓN

\begin{tabular}{ccl}
\hline $\begin{array}{c}\text { Exigencia mental } \\
(\mathrm{M})\end{array}$ & Baja(0)/Alta(5) & $\begin{array}{l}\text { ¿Cuánta actividad mental y perceptiva fue necesaria? (Por } \\
\text { ejemplo: pensar, decidir, calcular, recordar, buscar, investigar, } \\
\text { etc.). ¿Se trata de una tarea fácil o difícil, simple o compleja, } \\
\text { pesada o ligera? }\end{array}$ \\
\hline $\begin{array}{c}\text { Exigencia física } \\
(\mathrm{F})\end{array}$ & Baja/Alta & $\begin{array}{l}\text { ¿Cuánta actividad física fue necesaria? (Por ejemplo: empujar, } \\
\text { tirar, girar, pulsar, accionar, etc.) ¿Se trata de una tarea fácil ó } \\
\text { difícil, lenta o rápida, relajada o cansada? }\end{array}$ \\
\hline
\end{tabular}

\begin{tabular}{|c|c|c|}
\hline $\begin{array}{l}\text { Exigencia } \\
\text { temporal }(\mathrm{T})\end{array}$ & Baja/Alta & $\begin{array}{l}\text { ¿Cuánta presión de tiempo sintió, debido al ritmo al cual se } \\
\text { sucedían las tareas o los elementos de las tareas? ¿Era el ritmo } \\
\text { lento y pausado ó rápido y frenético? }\end{array}$ \\
\hline
\end{tabular}




\begin{tabular}{cll}
\hline Esfuerzo (E) & Bajo/Alto & $\begin{array}{l}\text { ¿En qué medida ha tenido que trabajar (física o mental mente) } \\
\text { para alcanzar su nivel de resultados? }\end{array}$ \\
\hline Rendimiento (R) & Bueno/Malo & $\begin{array}{l}\text { ¿Hasta qué punto cree que ha tenido éxito en los objetivos } \\
\text { establecidos por el investigador (o por vd. mism@)? ¿Cuál es } \\
\text { su grado de sastisfacción con su nivel de ejecución? }\end{array}$ \\
\hline $\begin{array}{c}\text { Nivel de } \\
\text { frustración (Fr) }\end{array}$ & Bajo/Alto & $\begin{array}{l}\text { Durante la tarea, en qué medida se ha sentido insegur@ } \\
\text { desalentad@, irritad@, tens@) o preocupad@ o por el } \\
\text { contrario, se ha sentido segur@, content@, relajad@ y } \\
\text { satisfech@? }\end{array}$ \\
\hline
\end{tabular}

En la fase de puntuación, las personas valoran la tarea que han realizado en cada una de las dimensiones marcando un punto en la escala que se les presenta. Cada escala está dividida en 20 intervalos iguales, y debe convertirse a una puntuación sobre 100 (Figura 1).

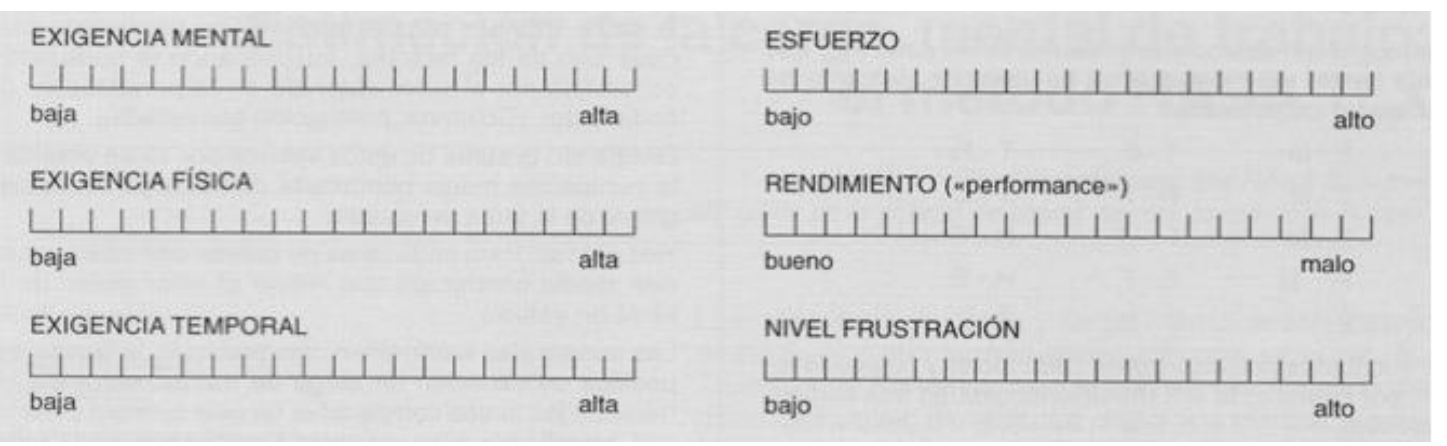

Figura 1. Fase de puntuación NASA-TLX.

Fuente: Llorca, J. (2009). Metodología de identificación y evaluación de la carga mental. Centro territorial de Seguridad y Salud en el Trabajo, Servicio de Especialidades Preventivas, Valencia.

Tras haberse rellenado la ponderación y la puntuación, se debe rellenar una tabla que considere las seis dimensiones y su peso obtenido en la ponderación sobre 5, la puntuación en la escala sobre 20, la puntuación convertida a una escala sobre 100 y la puntuación ponderada (peso multiplicado por la puntuación convertida). Finalmente se obtendrá un total y una media ponderada global de cada subescala que reflejará la importancia de cada uno de los factores como causantes de carga de trabajo y su importancia subjetiva en cada tarea. 
A la hora de interpretar las puntuaciones obtenidas, se considerará un nivel bajo de cada exigencia individual aquellas inferiores o iguales a 100, y alto las superiores. Para la suma total de puntuaciones que nos definirán un nivel de carga mental, las puntuaciones se consideran bajas por debajo o igual a 500, medias entre 500 y 1000 y altas por encima de 1000. El nivel medio ya supone un riesgo potencial que, de no desaparecer los factores asociados, puede evolucionar a alto, por lo que pueden interpretarse los resultados dividiéndolos en nivel bajo y nivel medio-alto.

Variables: Las variables estudiadas para llevar a cabo este trabajo son diversas, pero podemos clasificarlas en dependientes e independientes.

Las variables dependientes son aquellas obtenidas mediante el cuestionario NASA-TLX: exigencia mental, física, temporal, esfuerzo, rendimiento y nivel de frustración.

Como variables independientes, se han seleccionado una serie de datos sociodemográficos y laborales:

Edad: se trata de una variable numérica, cuantitativa, de razón y discreta. Se ha clasificado la edad en cuatro grandes grupos de forma que fuese una variable politómica ("25-35", “36-45", “46-55” y “>55” años) para, posteriormente dicotomizar los resultados en dos grandes grupos: " $\leq 45$ años” y “>45 años".

Sexo: variable categórica, cualitativa, nominal y dicotómica ("sexo masculino" y "sexo femenino").

Número de hijos: esta variable es categórica, cualitativa, nominal y politómica, por lo que las posibles respuestas son “0”, “1”, “2” y "3 o más”. Puede dicotomizarse en dos posibles respuestas ("hijos sí" e "hijos no").

Antigüedad en el puesto de trabajo: esta variable es categórica, cualitativa, ordinal y politómica. Se ha agrupado en cuatro grupos que son "1-5 años", "6-15 años”, "16-25 años” y “>25 años”. 
Tipo de contrato laboral: la variable es categórica, cualitativa, nominal y politómica, pues se han aceptado tres posibilidades ("fijo", "interno” y "sustitución”), pero puede dicotomizarse posteriormente en "contrato fijo" y "contrato temporal".

Tipo de turno laboral: variable categórica, cualitativa, nominal y dicotómica, pues existen solo dos posibilidades: el turno de 7 horas y el de 12 horas.

Procesamiento de los datos: Los datos obtenidos se han introducido en el programa Excel de Microsoft Office $2010 ®$ en forma de tabla, para posteriormente procesarlos en el programa de IBM SPSS ${ }^{2}$, en su versión 22.0 para poder llevar a cabo el análisis descriptivo y bivariante con las múltiples opciones que dicho programa ofrece.

En primer lugar se analizará de forma descriptiva cada variable obteniendo frecuencias, porcentajes y, en algunos casos, medias y desviaciones típicas. Los resultados se clasificarán en tablas para su fácil análisis y entendimiento.

Posteriormente, se analizará de forma bivariante cada uno de los cuestionarios con cada una de las variables sociodemográficas y laborales. Se empleará la prueba estadística Chi-cuadrado para estudiar la significación de cada una de estas relaciones bivariantes.

\section{Resultados}

Análisis descriptivo: La tasa de respuesta por parte de la población seleccionada en el estudio es del 100\%, ya que el dossier de cuestionarios se rellenó personalmente con cada uno de los profesionales que cumplían los requisitos. En la siguiente tabla (nํ 3) se exponen las características sociodemográficas y laborales de la muestra. 
Tabla 2. Características sociodemográficas y laborales de la muestra.

\begin{tabular}{|c|c|c|}
\hline & Variables & \\
\hline Edad & $\begin{array}{c}\mathrm{B} \pm \mathrm{DS} \\
\text { 25-35 años } \mathrm{n}(\%) \\
\text { 36-45 años } \mathrm{n}(\%) \\
46-55 \text { años } \mathrm{n}(\%) \\
>55 \text { años } \mathrm{n}(\%)\end{array}$ & $\begin{array}{c}43,40 \pm 11,57 \\
11(27,5 \%) \\
9(22,5 \%) \\
12(30 \%) \\
8(20 \%)\end{array}$ \\
\hline Sexo & $\begin{array}{l}\text { Femenino } \mathrm{n}(\%) \\
\text { Masculino } \mathrm{n}(\%)\end{array}$ & $\begin{array}{l}29(72,5 \%) \\
11(27,5 \%)\end{array}$ \\
\hline $\mathbf{N}^{\mathbf{o}}$ de hijos & $\begin{array}{c}0 \mathrm{n}(\%) \\
1 \mathrm{n}(\%) \\
2 \mathrm{n}(\%) \\
3 \text { o más n(\%) } \\
\text { Hijos sí/ Hijos no n(\%)* }\end{array}$ & $\begin{array}{c}15(37,5 \%) \\
11(27,5 \%) \\
10(25 \%) \\
4(10 \%) \\
25(62,5 \%) / 15(37,5 \%)^{*}\end{array}$ \\
\hline Antigüedad del puesto & $\begin{array}{l}\text { 1-5 años } \mathrm{n}(\%) \\
\text { 6-15 años } \mathrm{n}(\%) \\
\text { 16-25 años } \mathrm{n}(\%) \\
>25 \text { años } \mathrm{n}(\%)\end{array}$ & $\begin{array}{l}19(47,5 \%) \\
5(12,5 \%) \\
11(27,5 \%) \\
5(12,5 \%)\end{array}$ \\
\hline Contrato laboral & $\begin{array}{c}\text { Puesto fijo } \mathrm{n}(\%) \\
\text { Puesto interino } \mathrm{n}(\%) \\
\text { Sustitución } \mathrm{n}(\%) \\
\text { Puesto fijo/Puesto temporal } \mathrm{n}(\%)^{*}\end{array}$ & $\begin{array}{c}23(57,5 \%) \\
14(35 \%) \\
3(7,5 \%) \\
23(57,5 \%) / 17(42,5 \%)^{*}\end{array}$ \\
\hline Turno laboral & $\begin{array}{l}7 \text { horas } \mathrm{n}(\%) \\
12 \text { horas } \mathrm{n}(\%)\end{array}$ & $\begin{array}{l}20(50 \%) \\
20(50 \%)\end{array}$ \\
\hline
\end{tabular}

Leyenda:

- $\quad B \pm D S:$ media \pm desviación típica

- $\mathrm{n}$ : frecuencia

- $\%$ : porcentaje de casos

- $\quad$ * Variable dicotomizada

La edad media de la muestra es de 43,28 años, con una desviación típica de 11,49. El mínimo de edad es de 25 años y máximo de 61 años, pero destaca el grupo de personas de 46 a 55 años, que representan el $30 \%$ de la muestra ( $n=12)$, seguidos de las de 25-35 años que son un 27,5\% ( $n=11)$, el grupo de entre 36 y 45 años que conforman el 22,5\% (n=9) y por último, aquellas que superan los 55 años que tan solo representan un $20 \%(n=8)$.

Hay una notable representación femenina en la muestra, pues el 72,5\% de los encuestados fueron mujeres $(n=29)$, frente al $27,5 \%$ que eran hombres $(n=11)$. 
En la variable sociodemográfica "Número de hijos" nos encontramos con una tendencia decreciente en cuanto a porcentaje de muestra a medida que crece el $\mathrm{n}$ o de hijos. El grupo más grande es el formado por aquellos que no tienen hijos, pues representan un $37,5 \%$ de la muestra $(n=15)$, seguido de aquellos que tienen uno $(27,5 \%)$ y dos hijos (25\%) respectivamente. Finalmente observamos un pequeño grupo que constituye el $10 \%$ y tiene 3 o más hijos $(n=4)$. Al dicotomizar esta variable en tener o no hijos, tenemos un grupo del 62,5\% $(n=25)$ que sí los tiene, frente al $37,5 \%$ que no tiene $(n=15)$.

En cuanto a las variables de carácter laboral, se puede observar que un alto porcentaje de los encuestados $(47,5 \%, n=19)$ no superaban los 5 años de antigüedad en su puesto de trabajo, frente a los otros tres grupos. Aquellas personas que llevan entre 6 y 15 años en su puesto de trabajo tan solo representan un 12,5\% (n=5), a diferencia de aquellas que cuentan con una antigüedad de entre 16 y 25 años, que conforman un $27,5 \%$ de la muestra $(n=11)$. Por último, encontramos otro pequeño grupo de un $12,5 \%(n=5)$ cuya longevidad en el puesto de trabajo supera los 25 años.

Podemos apreciar que en el tipo de contrato laboral destaca el grupo de personas con contrato fijo, que son un $57,5 \%(n=23)$, inmediatamente seguidos de aquellos con un contrato de interinidad que son un $35 \%(n=14)$ y, por último, de aquellos que están haciendo una sustitución que son un 7,5\% de la muestra total $(\mathrm{n}=3)$. El tipo de turno (7 horas o 12 horas) divide a la muestra en dos mitades iguales (50\%-50\%), pues formaba parte de la estrategia de selección de ésta.

La tabla 4 contiene las variables que el cuestionario NASA-TLX emplea para medir la carga mental de las personas en su puesto de trabajo.

Tabla 3. Resultado del cuestionario NASA-TLX.

\begin{tabular}{lcc} 
Variables & \\
\hline Exigencias mentales & $\mathrm{B} \pm \mathrm{DS}$ & $234,38 \pm 137,79$ \\
& Nivel bajo n $(\%)$ & $9(22,5 \%)$ \\
& Nivel medio-alto n $(\%)$ & $31(77,5 \%)$ \\
\hline
\end{tabular}




\begin{tabular}{|c|c|c|}
\hline Exigencias físicas & $\begin{array}{c}\mathrm{B} \pm \mathrm{DS} \\
\text { Nivel bajo } \mathrm{n}(\%) \\
\text { Nivel medio-alto } \mathrm{n}(\%)\end{array}$ & $\begin{array}{c}110,63 \pm 126,84 \\
27(67,5 \%) \\
13(32,5 \%)\end{array}$ \\
\hline Exigencias temporales & $\begin{array}{c}\mathrm{B} \pm \mathrm{DS} \\
\text { Nivel bajo } \mathrm{n}(\%) \\
\text { Nivel medio-alto } \mathrm{n}(\%)\end{array}$ & $\begin{array}{c}226,75 \pm 117,98 \\
11(27,5 \%) \\
29(72,5 \%)\end{array}$ \\
\hline Rendimiento & $\begin{array}{c}\mathrm{B} \pm \mathrm{DS} \\
\text { Nivel bajo } \mathrm{n}(\%) \\
\text { Nivel medio-alto } \mathrm{n}(\%)\end{array}$ & $\begin{array}{c}133,38 \pm 84,41 \\
16(40 \%) \\
24(60 \%)\end{array}$ \\
\hline Esfuerzo & $\begin{array}{c}\mathrm{B} \pm \mathrm{DS} \\
\text { Nivel bajo }(\%) \\
\text { Nivel medio-alto } \mathrm{n}(\%)\end{array}$ & $\begin{array}{c}154,5 \pm 100,78 \\
15(37,5 \%) \\
25(62,5 \%)\end{array}$ \\
\hline Frustración & $\begin{array}{c}\mathrm{B} \pm \mathrm{DS} \\
\text { Nivel bajo } \mathrm{n}(\%) \\
\text { Nivel medio-alto } \mathrm{n}(\%)\end{array}$ & $\begin{array}{c}100,38 \pm 113,43 \\
23(57,5 \%) \\
17(42,5 \%)\end{array}$ \\
\hline Carga Mental & $\begin{array}{c}\mathrm{B} \pm \mathrm{DS} \\
\text { Nivel bajo } \mathrm{n}(\%) \\
\text { Nivel medio } \mathrm{n}(\%) \\
\text { Nivel alto } \mathrm{n}(\%) \\
\text { Nivel bajo/Nivel medio-alto } \mathrm{n}(\%) *\end{array}$ & $\begin{array}{c}960 \pm 201,5 \\
1(2,5 \%) \\
22(55 \%) \\
17(42,5 \%) \\
1(2,5 \%) / 39(97,5 \%)^{*}\end{array}$ \\
\hline
\end{tabular}

Fuente: elaboración propia. 2017.

Como se puede observar, en la primera variable "Exigencias mentales", existe una gran disparidad de valores al hacer la media, pues es de 234,38 y la desviación típica es de 137,79, de hecho, el rango de valores va desde un mínimo de 0 hasta un máximo de 450. Los datos están clasificados en dos niveles, el bajo tiene un porcentaje de $22,5 \%(n=9)$ y el medio-alto, del $77,5 \%(n=31)$.

En cuanto a "Exigencias físicas", la situación es similar, la media es de 110,63 $\pm 126,84$ de desviación típica, con mínimo y máximo de 0 y 500 respectivamente. En el nivel bajo podemos encontrar un $67,5 \%$ de la muestra $(n=27)$ y en el nivel medioalto al $32,5 \%$ restante $(n=13)$.

En la variable "Exigencias temporales" los datos parecen tener una tendencia más centralizada que en las dos anteriores, pues la media es de 226,75 $\pm 117,98$ de 
desviación típica, siendo el mínimo 0, y el máximo 450. Un 27,5\% conforma el nivel bajo $(n=11)$ frente a un $72,5 \%$ que lo hace con el nivel medio-alto $(n=29)$.

Las variables de "Rendimiento" y "Esfuerzo" tienen resultados similares, con medias de 133,38 \pm 84,41 y 154,5 $\pm 100,78$ de desviación típica respectivamente. En el nivel bajo de carga mental por rendimiento encontramos a un $40 \%$ de la muestra $(n=16)$ y en el medio-alto al $60 \%$ restante $(n=24)$. Por otro lado, en esfuerzo tenemos a un $37,5 \%$ en la categoría baja $(n=15)$ y a un $62,5 \%$ en la medio-alta $(n=25)$.

Se observan niveles bajos de "Frustración", pues esta categoría representa el $57,5 \%$ del total $(n=23)$ frente al $42,5 \%$ con niveles medios-altos $(n=17)$. La media es de 100,38 con una desviación de 113,43 puntos. En el rango de valores va de 0 hasta 380.

Estas variables en sí mismas tienen un sentido, pero cuando se tienen en cuenta en conjunto, nos permiten medir la "Carga mental". La media de valores obtenidos es de $960 \pm 201,5$ de desviación típica, con un mínimo de 470 y un máximo de 1250 , lo cual nos indica un nivel medio de carga mental. Los resultados se dividen en tres categorías, siendo la primera de ellas el nivel bajo, que tan solo es del 2,5\% $(n=1)$, seguido del nivel medio con un $55 \%(n=22)$ y del nivel alto con un $42,5 \%$ $(\mathrm{n}=17)$. Al dicotomizar la variable en "nivel bajo" y "nivel medio-alto" tenemos un porcentaje de 2,5\% (n=1) y 97,5\% (n=39) respectivamente.

Análisis bivariante: Mediante el programa IBM SPSS Stadistics $®$ se ha llevado a cabo un análisis de los resultados del NASA-TLX en relación con las variables sociodemográficas y laborales. Antes de ello, ha sido primordial hacer una prueba de normalidad de las variables, que en este caso ha sido Shaphiro Wilk debido al número inferior a 50 de casos. El resultado obtenido es de $\mathrm{p}<0,05$ en todas las variables, por lo que no se ajustan a la normalidad. Convirtiéndolas todas a variables cualitativas, podemos utilizar la prueba Chi-cuadrado. 


\section{Puntuación del cuestionario NASA-TLX en relación con las variables sociodemográficas y labores:}

Edad: en la tabla 5 se han calculado las medias de las puntuaciones del cuestionario NASA-TLX en función de la edad de los profesionales de Enfermería que forman la muestra de estudio, agrupados en 4 grupos de edad. Para el grupo más joven, con edades comprendidas entre los 25 y los 35 años, el nivel de carga mental global de 990,46 $\pm 279,32$. El siguiente grupo de edad, formado por los profesionales de Enfermería de entre 36 y 45 años, tiene puntuación ligeramente inferior de carga mental respecto al grupo anterior, con una media de puntuación global de $950 \pm 149,15$. El grupo comprendido entre las edades de 46 a 55 años, tuvo una media de puntuación para la carga mental de 879,17 $\pm 235,63$. Por último, los mayores de 55 años tuvieron una media de puntuación de 975,63 $\pm 199,63$. Por tanto, los niveles de carga mental por grupos de edad, son mayores a partir de los 55 años, así como en las edades más tempranas de la muestra (25-35 años).

Sexo: a la hora de comparar a los hombres y las mujeres (tabla 6), obtenemos que la carga mental de las mujeres resulta ser mayor con 989,14 $\pm 204,32$ puntos de desviación típica, versus los 883,18 \pm 180,32 de los hombres. No obstante, ninguna de las comparaciones de medias es estadísticamente significativa, pues en ningún caso p es igual o inferior a 0,05.

Número de hijos: en la tabla 7 se presentan las diferencias del cuestionario NASA-TLX en función de la descendencia que compone la estructura familiar de los profesionales de Enfermería de la investigación. El nivel general de carga mental más alto lo dan profesionales de Enfermería sin hijos $(1007,33 \pm 172,74)$, pero de nuevo la $\mathrm{p}$ es mayor de 0,05 y no se trata de un resultado estadísticamente significativo. 
Antigüedad del puesto de trabajo: al analizar el nivel de carga mental en función de la antigüedad del puesto de trabajo (tabla 8), nos encontramos con que es mayor para aquellos que llevan más de 25 años en su puesto de trabajo $(1004,00$ $\pm 242,52)$, seguidos de los más nuevos $(993,95 \pm 199,42)$, siendo el grupo que percibe menor carga mental aquel que lleva entre 6 y 15 años en su puesto de trabajo $(816,00 \pm 272,11)$. La p obtenida mediante la prueba estadística Chicuadrado nos ha dado un resultado de 0,001 , por lo que estos resultados son significativos.

Tipo de contrato laboral: la puntuación global que nos permite conocer la carga mental de aquellos con contrato temporal arroja una media de 998,53 \pm 206,34 de desviación típica, a diferencia de los que tienen un contrato fijo, cuya media es de 931,52 \pm 197,54 puntos (Tabla 9).

Tipo de turno laboral: en la tabla 10 se puede observar que no existen diferencias significativas entre las medias para el turno de 7 horas y para el $12 \mathrm{~h}$, siendo ligeramente superior en el turno de 12 horas $(972 \pm 185,10$ frente a $948 \pm$ $220,86)$, pero no es un dato estadísticamente significativo.

Tabla 4. Puntuación del cuestionario NASA-TLX por grupo de edad.

\begin{tabular}{ccccccc}
\hline Componentes & Edad & $25-35$ & $36-45$ & $46-55$ & $>55$ & $\mathrm{p}$ \\
& & & & & & \\
\hline Puntuación global & Nivel de carga & & & & & \\
de NASA-TLX & mental & $990,46 \pm$ & $950 \pm$ & $879,17 \pm$ & $975,63 \pm$ & 0,50 \\
& B \pm DS & 279,32 & 149,15 & 235,63 & 199,63 & \\
\hline
\end{tabular}


Tabla 5. Resultado de cuestionario NASA-TLX por sexo.

\begin{tabular}{ccccc}
\hline Componentes & Sexo & Masculino & Femenino & p \\
\hline $\begin{array}{c}\text { Puntuación global de } \\
\text { NASA-TLX }\end{array}$ & $\begin{array}{c}\text { Nivel de carga mental } \\
\text { B } \pm \text { DS }\end{array}$ & $883,18 \pm 180,32$ & $989,14 \pm 204,32$ & 0,53 \\
\hline
\end{tabular}

Fuente: elaboración propia. 2017.

Tabla 6. Resultado de cuestionario NASA-TLX por tenencia de hijos.

\begin{tabular}{cccc}
\hline Componentes & Hijos & Hijos sí & Hijos no \\
\hline $\begin{array}{c}\text { Puntuación global de } \\
\text { NASA-TLX }\end{array}$ & $\begin{array}{c}\text { Nivel de carga } \\
\text { mental } \\
\text { B } \pm \text { DS }\end{array}$ & $931,60 \pm 215,26$ & $1007,33 \pm 172,74$ \\
\hline
\end{tabular}

Fuente: elaboración propia. 2017.

Tabla 7. Resultado del cuestionario NASA-TLX por antigüedad del puesto.

\begin{tabular}{lcccccc}
\hline Componentes & Antigüedad del puesto & $1-5$ & $6-15$ & $16-25$ & $>25$ & $\mathrm{p}$ \\
\hline $\begin{array}{c}\text { Puntuación global } \\
\text { de NASA-TLX }\end{array}$ & $\begin{array}{c}\text { Nivel de carga mental } \\
\text { B } \mathbf{D S}\end{array}$ & $\begin{array}{c}993,95 \pm \\
199,42\end{array}$ & $\begin{array}{c}816,00 \pm \\
272,11\end{array}$ & $\begin{array}{c}946,82 \\
\pm 141,11\end{array}$ & $\begin{array}{c}1004,00 \pm \\
242,52\end{array}$ & 0,001 \\
& & & & & & \\
\hline
\end{tabular}

Fuente: elaboración propia. 2017.

Tabla 8. Puntuación del cuestionario NASA-TLX por tipo de contrato laboral.

\begin{tabular}{ccccc}
\hline Componentes & $\begin{array}{c}\text { Tipo de contrato } \\
\text { laboral }\end{array}$ & Puesto fijo & Puesto no fijo & p \\
\hline $\begin{array}{c}\text { Puntuación global de } \\
\text { NASA-TLX }\end{array}$ & $\begin{array}{c}\text { Nivel de carga mental } \\
\text { B } \pm \text { DS }\end{array}$ & $931,52 \pm 197,54$ & $998,53 \pm 206,34$ & 0,38 \\
& & & \\
\hline
\end{tabular}

Fuente: elaboración propia. 2017. 
Tabla 9. Puntuación del cuestionario NASA-TLX por tipo de turno laboral.

\begin{tabular}{ccccc}
\hline Componentes & Tipo de turno & 7 horas & 12 horas & p \\
\hline $\begin{array}{c}\text { Puntuación global de } \\
\text { NASA-TLX }\end{array}$ & $\begin{array}{c}\text { Nivel de carga } \\
\text { mental } \\
\text { B } \mathbf{D S}\end{array}$ & $948 \pm 220,86$ & $972 \pm 185,10$ & 0,31 \\
& & & \\
\hline
\end{tabular}

Fuente: elaboración propia. 2017.

\section{Discusión}

El propósito de este estudio era medir la carga mental de los profesionales de Enfermería de varias unidades de un hospital determinado y la relación con su turno de trabajo; además de conocer si esta relación era mayor que la establecida con otros factores sociodemográficos y laborales.

Los resultados obtenidos en el presente estudio, muestran que un 55\% de los investigados tienen un nivel de carga mental media y más del 40\%, un nivel alto. Los aspectos más resaltados por los enfermeros son las exigencias mentales y temporales, al igual que en el estudio de Flores et al (2014), a pesar de que en su estudio un $75 \%$ de la muestra presenta niveles medios de carga mental y un $25 \%$, niveles altos. Sin embargo estudios como el de Barrera et al (2014) dan más peso a parámetros como el esfuerzo percibido acompañado de las exigencias temporales.

La primera hipótesis que se planteó al inicio de este estudio fue la existencia de profesionales agotados mentalmente. Observando los resultados del cuestionario NASA-TLX, podemos afirmar que sí existen profesionales de Enfermería agotados a nivel mental, presentando un nivel medio-alto de carga mental, dato similar a otros estudios como los de Albadalejo et al (2004) y Sanjuan et al (2014).

Otra de las hipótesis era que existe una relación positiva entre los turnos laborales más largos (de 12 horas) y mayores niveles carga mental. Los resultados de esta investigación nos revelan que no existe una relación estadísticamente 
significativa entre el turno laboral y la carga mental. Esto contrasta con los resultados de Albadalejo et al (2004), quien encuentra diferencias significativas para la variable "turno" detectando mayor Burnout en los turnos rotatorios de 12 horas. Otros autores como Deschamps et al (2011) y Melita et al (2008) han podido establecer esta relación también en sus estudios. Además, González et al (2005) y Knauth (1993) han observado una influencia de turnos largos en el incremento de la carga mental y los niveles de estrés de los profesionales de Enfermería.

Una última hipótesis planteada era que pudiera existir una relación positiva entre otras variables sociodemográficas y laborales como la edad, el sexo, la tenencia de hijos, la antigüedad del puesto de trabajo y el tipo de contrato con la carga mental, aspecto que sí podemos afirmar en mayor o menor medida. Díaz (2017) ha podido establecer una relación positiva entre carga mental y edad, sin embargo González et al (2005) no encuentra diferencias significativas.

Por otro lado, un estudio de Ceballos et al (2014) sí encuentra diferencias significativas para el sexo femenino, observando mayores niveles de dicha carga.

En los resultados obtenidos, influye en la carga mental llevar más de 25 años en el mismo puesto de trabajo, tener entre 25 y 35 años, tener hijos y tener un contrato temporal. Los resultados de nuestro estudio son similares a los de Díaz (2017), que también encuentra una relación positiva entre la antigüedad del puesto de trabajo y esta variable. Sin embargo, estos datos poco tienen que ver con los de Atance (1997) y Marrero y Grau (2005), que no han encontrado diferencias significativas según la tenencia de hijos. Quizá haya que analizar más características familiares como el estado civil, el apoyo por parte de otros familiares, la edad de los hijos o la ocupación laboral de la pareja.

Estudios como el de Quiroz y Saco (2003), De França et al (2012) y Ortega et al (2007), en cambio, sí observan una influencia significativa de la temporalidad del contrato.

\section{Conclusiones}


Teniendo en cuenta la alta tasa de profesionales de Enfermería en riesgo o sufriendo altas cargas mentales, es imprescindible no solo ampliar horizontes en cuanto a la investigación de este tema, sino buscar consenso entre autoridades y sindicatos que modifiquen las condiciones laborales de estos profesionales en función de los factores que parecen desencadenar estos efectos negativos en su estado mental y, por supuesto, emocional, pues las repercusiones alcanzan el plano de la salud física. Altos niveles de carga mental pueden derivar en problemas cardiovasculares, digestivos y neurológicos, entre otros (Knauth, 2001; Tovalin, 2005; Feo, 2007; Celorrio, 2015).

Por tanto, conocer la prevalencia de la carga mental puede ser útil para diseñar estrategias de prevención y reducción del impacto del problema, produciendo, a su vez, una mejoría en los servicios prestados por los profesionales de Enfermería.

\section{Limitaciones del estudio}

Durante el desarrollo de este estudio, se han detectado varias limitaciones, destacando entre ellas el reducido tamaño de la muestra y la diferencia proporcional entre hombres y mujeres. Además, existe muy poca bibliografía acerca de carga mental medida con cuestionarios tipo NASA-TLX en la profesión enfermera.

\section{Bibliografía}


Albadalejo, R., Villanueva, R., Ortega, P., Astasio, P., Calle, M. y Domínguez, V. (2004). Síndrome de burnout en el personal de enfermería de un hospital de Madrid. Rev Esp Salud Publica, 78(4).

Atance, J. (1997). Aspectos epidemiológicos del Síndrome de Burnout en personal sanitario. Rev Esp Salud Publica, 71(3).

Barrera, R., Díaz, L., Busto, J., Romero, L. y Domínguez Ramírez, O. (2014). Realización de una evaluación de un sistema de interacción físico hombre-robot con base en el protocolo NASA-TLX. Rev y Boletines científicos, 3(5).

Ceballos, P., Valenzuela, S. y Paravic, T. (2014). Factores de riesgos psicosociales en el trabajo: genero y enfermeria. Avances en Enfermería., 32(2), 271-279.

Celorrio, C. (2015). Minerva investiga. Recuperado el 27 de Noviembre de 2016, de http://www.index-f.com/blogminerva/?p=932

De Cássia, D., Almeida, L., Oliveira, A. y Do Campo, M. (2014). Carga horaria de trabajo de los enfermeros y su relación con las reacciones fisiológicas de estrés. Rev. Latino-Am. Enfermagem, 22(6), 959-965.

De França, F., Ferrarri, R., Ferrari, D. y Alves, E. (2012). Burnout y aspectos laborales del personal de enfermería de dos hospitales de medio portea. Rev Latino-Am. Enfermagem, 20(5).

Deschamps, A., Olivares, S., De la Rosa, K. y Asunsolo, A. (2011). Influencia de los turnos de trabajo y las guardias nocturnas en la aparición del Síndrome del Burnout en médicos y enfermeras. Med segur Trab, 57(224), 224-241.

Díaz, W. (2017). Sobrecarga laboral asociado a Síndrome de Burnout en personal de salud de emergencia de un hospital de Trujillo. Facultad de Medicina Humana Antenor Orrego.

Feo, J. (2007). Influencia del trabajo por turnos en la salud y la vida cotidiana. Pontífica Universidad Javeriana. Facultad de Enfermería y Medicina. 
Flores, D., Vega, V., Del Río, C. y Zavala, D. (2014). Ocuparse del bienestar de los profesionales de la salud: un desafío pendiente. Revista Chilena de Terapia Ocupacional, 14(1).

González, J., Moreno, B., Garrosa, E. y López, A. (2005). Carga mental y fatiga en servicios especiales de enfermería. Rev. Latinoam. Psicol., 37(3).

Knauth, P. (2001). Horas de trabajo. Enciclop de salud y seguridad en el trabajo, $43(2)$.

Knauth, P. (1993). The design of shift systems. Ergonomics, 36, 15-28.

Llorca, J. (2009). Metodología de identificación y evaluación de la carga mental. Centro territorial de Seguridad y Salud en el Trabajo, Servicio de Especialidades Preventivas, Valencia.

Marrero, M. y Grau, J. (2005). Burnout syndrome in medical staff working in neonatal intensive care units. Psicología y Salud, 15(1), 25-32.

Melita, A., Cruz, M. y Manuel, J. (2008). Burnout en profesionales de enfermería que trabajan en centros asistenciales de la octava región, Chile. Ciencia. enferm., 14(2).

Moreno, M., Jeréz, J., Cabrera, S., Estrada, J. y López, A. (2013.). Turnos de 7 horas versus 12 horas en enfermería intensiva: vivir a contratiempo. Escuela Universitaria de Enfermería. Universidad de Barcelona., Enfermería Fundamental y medicoquirúrgica., L'Hospitalet de Llobregat (Barcelona).

Morin, E. (1970). La noción del sujeto. En E. Morin, El hombre y la muerte (págs. 110). Barcelona: Kairás.

NIOSH. (2016). Recuperado el 2 de Diciembre de 2016, de Centros para el Control y la Prevención de Enfermedades.: https://www.cdc.gov/spanish/niosh/topics/turnos.html

Ortega, M., Ortíz, G. y Coronel, P. (2007). Burnout en médicos y enfermeras y su relación con el constructo de personalidad resistente. Psicología y Salud, 17(1), 516. 
Quiroz, R. y Saco, S. (2003). Factores asociados al Síndrome Burnout en médicos y enfermeras del Hospital Nacional Sureste de Essalud del Cusco. Rev Semestral de la Facultad de Medicina Humana, 12(23), 11-22.

Sanjuan, L., Arrazola, O. y García, L. (2014). Prevalencia del Síndrome del Burnout en el personal de enfermería del Servicio de Cardiología, Neumología y Neurología del Hospital San Jorge de Huesca. Enfermería Global., 36, 253-264.

Sebastián, O. y Del Hoyo, M. (2002). La carga mental de trabajo. Documentos divulgativos. Ministerio de trabajo y asuntos sociales.

Tovalin, H. (2005). Rotación de turnos, fatiga y trastornos del sueño en un grupo de trabajadores industriales. Rev Cub de salud y Trabajo, 61, 27-31.

WHO. (2016). Recuperado el 22 de Noviembre de 2016, de Organización Mundial de la Salud. Preguntas más frecuentes.: http://www.who.int/suggestions/faq/es/ 